\title{
Engineering Microenvironment to Study Tumour Behaviour
}

\author{
José María Ayuso, Iñaki Ochoa, Luis Fernández \\ Group of Structural Mechanics and Materials Modelling (GEMM) \\ Instituto de Investigación en Ingeniería de Aragón (I3A) \\ Universidad de Zaragoza, Mariano Esquillor s/n, 50018, Zaragoza, Spain. \\ Tel. +34-976762707, e-mail: josayuso@unizar.es
}

\section{Abstract}

Microtechnologies are arising as powerful tools to recreate complex biological scenarios, like tumour development and invasion.

\section{Introduction}

Cancer stands as the first mortality cause in developed countries, being capable of affecting mostly any tissue and spread throughout the organism [1]. As research progress, we are more aware of the cancer complexity. Nowadays, cancer is seen as a multifactorial process, in which tumour cells coexist with the stromal cells (non-tumour cells) and interact with the surrounding tissue. As a result, a very complex microenvironment is frequently observed, involving complex mechanisms such as cell cross-talk, hypoxia (low oxygen concentration) or nutrient starvation [2]. Recent reports highlight the key role of this tumour microenvironment on patient prognosis and drug response. As an example, glioblastoma (GBM) is the most common and lethal brain tumour [3], and its aggressiveness seems to be linked to this hypoxic and starving microenviront [4]. Recently, microfabrication and microfluidics have arisen as promising technologies for the development of high-performance cell culture systems [5].

\section{Materials and methods}

\section{Microfabrication}

SU-8 based microdevices (Fig 1A) were fabricated using soft lithography techniques in the clean room located in the Instituto de Nanociencia de Aragon (INA).

\section{Cell culture}

Cells were routinely cultured in traditional Petri dishes with culture media (DMEM supplemented with $10 \%$ foetal bovine serum [FBS]).

\section{Microscopy}

Microscopy images were taken in a Nikon Eclipse Ti microscope equipped with a C1 modular confocal microscope system.

\section{Results}

\section{Liquid confinement and cell viability}

The presented microdevices have been designed with a special geometry that allows liquid confinement in the different microchambers (Fig 1B). Cells have been cultured within the microdevices in a 3D hydrogel, showing high cell viability (Fig 1C). This patterning capacity allowed the generation of spatially organized co-cultures (Fig 1D).

\section{Migration under nutrient starvation}

Enabling medium flow through one lateral microchamber, whereas the other remained closed, cell metabolism created a nutrient starvation in the central microchamber. When GBM cells were cultured under this nutrient starvation, a strong migratory response was observed, (Fig 2A). On the other hand, when both lateral microchambers remained opened, GBM distribution was contant (Fig 2B).

Oxygen measurement

Using hypoxia-induced fluorescent compounds, oxygen consumption within the 3D microenvironment could be studied (Fig 3A and B). As cell culture time increases, oxygen profile was monitored in real time, recreating the hypoxia gradient that is observed during tumour evolution in patients.

\section{Conclusions}


In the presented work, microtechnologies have been applied to the study of biological problems, like tumour development. Under nutrient starvation it is shown how GBM cell triggered a migratory response to the nutrient source.

\section{References}

[1]. JEMAL, et al. Global cancer statistics. CA: A Cancer Journal for Clinicians, Mar-Apr 2011, 61(2), 69-90.

[2]. CASEY, S.C., et al. Cancer prevention and therapy through the modulation of the tumor microenvironment. Seminars in
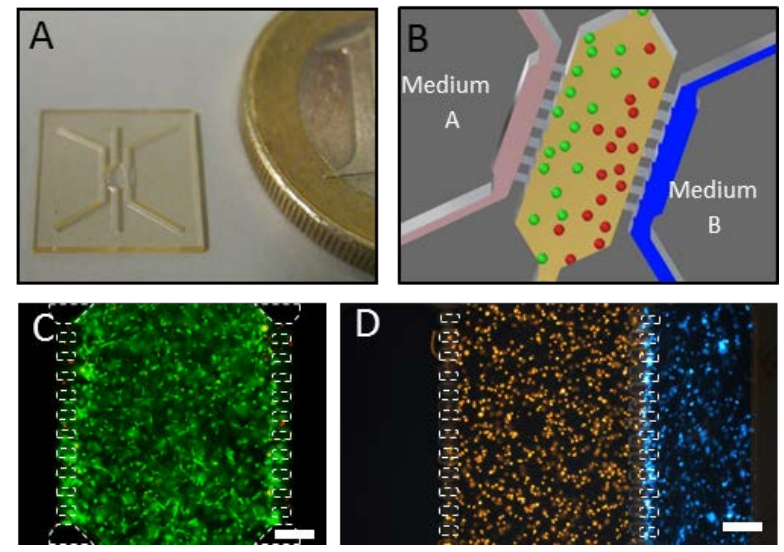

Cancer Biology [on line]. 2015, Apr 10. [consulted: 7 may 2015]. Avalaible from: doi:10.1016/j.semcancer.2015.02.007.

[3]. BRAT, D.J. Glioblastoma: Biology, Genetics, and Behavior. In: American Society of Clinical Oncology educational book. Alexandria: American Society of Clinical Oncology, 2011, pp. 102-107.

[4]. BRAT, D.J., et al. Pseudopalisades in glioblastoma are hypoxic, express extracellular matrix proteases, and are formed by an actively migrating cell population. Cancer Research. 2004, 64(3), 920-927.

[5]. SACKMANN, E. K., A. L. FULTON and D. J. BEEBE. The present and future role of microfluidics in biomedical research. Nature. 2014, 507(7491), 181-189.

Figure 1. A) Fabricated SU-8 based microdevice. B) Scheme of the microdevice internal geometry. C) Cell viability within a 3D collagen hydrogel confined in the central microchamber. Viable cells are shown in green whereas dead ones are in red. D) Different cell populations can be confined in the microdevices. Scale bar is $200 \mu \mathrm{m}$.
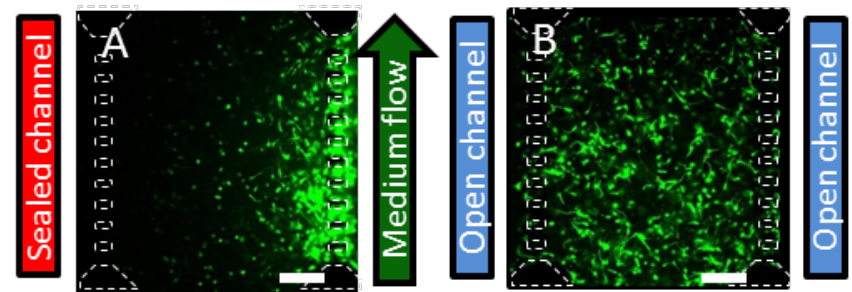

Figure 2 3D GBM cell culture under nutrient starvation. A) One lateral microchamber was closed whereas continuous medium flow was enabled in the other one. Cell metabolism created a nutrient starvation and after nine days in culture, GBM cells were concentrated nearby the perfused microchamber. B) When both lateral microchamber remained opened and refreshed daily, no migration was observed. $\quad$ Scale $\quad$ bar $\quad$ is $\quad 200 \quad \mu \mathrm{m}$
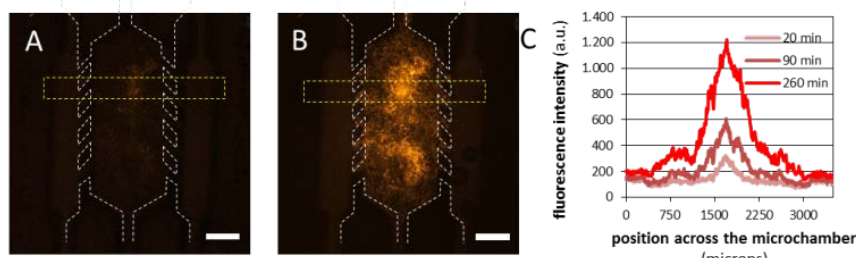

Figure 3. Hypoxia-induced fluorescent compounds were used to monitor in real time oxygen profile evolution due to cell metabolism. A) Hypoxia-induced fluorescent after $20 \mathrm{~min}$ in cell culture. B) Hypoxia-induced fluorescent after $260 \mathrm{~min}$ in cell culture. C) Graph shows oxygen profile evolution across the microchamber (region delimited in yellow in the images). Scale bar is $1 \mathrm{~mm}$. 